\title{
Inversão 1D de Dados Sintéticos de Um Dipolo Magnético Vertical Utilizando Os Regularizadores de Suavidade Global e Minimum Gradient Support
}

\author{
Silva,H.T.A,UFOPA; Piedade,A.A.,UFOPA
}

Copyright 2018, SBGf - Sociedade Brasileira de Geofísica

Este texto foi preparado para apresentação no VIII Simpósio Brasileiro de Geofísica, em Salinópolis, Pará, Brazil, de 18 a 20 de setembro de 2018. Seu conteúdo foi Revisado Pelo comitê técnico do VIII Simpósio Brasileiro de Geofísica, mas não representa a opinião da SBGf, seus sócios ou membros. A reprodução total ou parcial do conteúdo desse testo para fins comerciais sem 0 consentimento, por escrito, da Sociedade Brasileira de Geofísica é priobída.

\section{Resumo}

Neste presente tabalho será feita a modelagem e inversão de dados sintéticos de um dipolo magnético vertical em um meio estratificado 1D, que é uma fonte comum para alguns métodos eletromagnéticos. Será desenvolvido um algorítimo de inversão não linear utilizando o método de Gauss-Newton, com a técnica de Marquardt para garantir a convergência do processo iterativo de inversão. Com o instuito de tornar o problema inverso bem posto, eliminando a ambiguide das soluções no problema inverso, serão utilizados os regularizadores de Suavidade Global (SG) e Minimum Gradient Support (MGS). Será feito um estudo para identificar camadas alvos mais resistivas e mais condutívas que o seu meio encaixante, utilizando os dois regularizadores anteriormente mencionados.

\section{Introdução}

Na geofísica é comum o uso de métodos eletromagnéticos de efeitos indutivos na investigação de ambientes geológicos, por meio de suas propriedades elétricas. Estes acabam, muitas vezes, sendo uma maneira prática e acessível para esse fim. A inversão geofísica é uma poderosa ferramenta na interpretação de dados geofísicos, sendo uma das técnicas mais usadas na interpretação de dados de vários métodos.

Dentre os métodos eletromagnéticos indutivos, que trabalham com dados no domínio da frequência, há aqueles que utilizam como fontes de campos, bem como receptores, bobinas (loops) circulares ou não, que podem ter vários metros de diâmetro como o caso, por exemplo, do Método Eletromagnético a Multi Frequência (EMMF) (Piedade and Régis, 2014) ou alguns poucos metros como o método Slingram (Philip Kearey, 2009). Com o intuito de simular levantamentos desses métodos faremos a modelagem e a invers ão de dados de um dipolo magnético vertical (DMV), para um meio constituído de camadas plano paralelas, cujas propriedades físicas variam apenas verticalmente (meio 1D).

A modelagem do DMV será feita considerando, como fontes de campo, bobinas circulares de raio muito inferior a distância do mínimo offset fonte-receptor, seguindo a metodologia descrita por Rijo (2004). Na inversão, utilizaremos o método de Gauss-Newton com a estratégia de Marquardt (Marquardt, 1963), para inversão não linear.
O problema inverso por ser mal posto, incluiremos ao algoritmo de inversão os vínculos de Suavidade Global (SG ) e Minimum Gradient Support ( MGS ), afim de tornar o nosso problema bem posto e diminuir a ambiguidade em nossos modelos de interpretação.

\section{Metodologia}

\section{Fonte Dipolar Magnética}

O dipolo magnético vertical é constituído de uma bobina de raio muito pequeno, se comparado a distância entre o centro da bobina e o ponto de observação. A metodologia usada para modelar os dados dessa fonte, para um meio de camadas, será a descrita em Rijo (2004), onde serão medidos em várias posições de receptores e para várias frequências o campo

$$
\bar{H}_{r}=\frac{H_{r}(r, w)}{H_{z}^{0}(r)}
$$

onde $H_{r}$ é o campo magnético radial da bobina, constituído em um meio estratificado, e $H_{z}^{0}$ o componente vertical de um dipolo magnètico no vácuo, de momento magnético $m$, dado pela equação

$$
H_{z}^{0}(r)=-\frac{m}{4 \pi r^{3}} .
$$

Esta normalização é utilizada para minimizar o efeito do decaimento geométrico do campo. Os dados utilizados na inversão serão as partes real e imaginária do campo $\bar{H}_{r}$, para algumas posições de receptores e uma determinada faixa de frequência.

O método de Gauss-Newton, com a Estratégia de Marquardt, para inversão não linear

Dada as observações geofísicas contidas em um vetor $\mathbf{d}^{o}=\left[d_{1}^{o}, d_{2}^{o}, \ldots, d_{M}^{o}\right]$, e ainda uma função vetorial (modelo matemático), que será considerado não linear, $\mathbf{F}(\mathbf{P})=$ $\left[f_{1}(\mathbf{P}), \ldots, f_{N}(\mathbf{P})\right]$, que é função da frequência $\omega$, das posições de medida $(x, y, z)$, e ainda de um conjunto de parâmetros geofísicos $\mathbf{P}$, pode-se estabelecer a igualdade

$$
\mathbf{d}^{o}=\mathbf{F}(\mathbf{P}) \text {. }
$$

Com o objetivo de encontrar $\mathbf{P}$ que satisfaça (3), define-se o funcional de ajuste entre os dados observados $\mathbf{d}^{o}$ e os estimados por $\mathbf{F}(\mathbf{P})$, utilizando a equação

$$
\phi_{d}(\mathbf{P})=\sum_{i=1}^{M}\left(d_{i}^{o}-f_{i}(\mathbf{P})\right)^{2},
$$

em que $M$ representa o número de observações, $d_{i}^{o}$ a i-ésima componente do vetor de observações e $f_{i}(\mathbf{P})$ 
a i-ésima componente da função vetorial que reproduz os dados. Na forma matricial pode-se representar este funcional como a norma Euclidiana ,ao quadrado, do vetor de diferênças entre as observações e a função vetorial, ou seja,

$$
\phi_{d}(\mathbf{P})=\left\|\mathbf{d}^{o}-\mathbf{F}(\mathbf{P})\right\|^{2} .
$$

O problema de encontrar $\mathbf{P}$ que minimize 5 é mal-posto, de modo que para torná-lo bem-posto, será utilizado um funcional regularizador, definido aqui genericamente por $\phi_{r}(\mathbf{P})$, que insere informações em relação aos parâmetros $\mathbf{P}$ que se quer estimar. Com isso, o problema a ser resolvido será construído da seguinte forma

$$
\left\{\begin{array}{c}
\text { Minimizar } \phi_{r}(\mathbf{P}), \\
\text { Sujeito a }\|\mathbf{Y}-\mathbf{F}(\mathbf{P})\|^{2}=\delta,
\end{array}\right.
$$

em que $\delta$ representa um erro associado ao modelo matemático não ajustar perfeitamente os dados, devido aos dados conterem ruídos instrumentais ou de ambiente. Resolver este problema significa minimizar o funcional, denominado função objetivo, descrito pela seguinte expressão

$$
\phi_{\alpha}(\mathbf{P})=\phi_{d}(\mathbf{P})+\alpha \phi_{r}(\mathbf{P}),
$$

em que $\alpha$ um escalar positivo, chamado de parâmetro de regularização, que controla a importância da informação inserida pelo funcional regularizador ao processo de inversão.

Fazendo uma aproximação de 7 por uma série de Taylor até segunda ordem é possível encontrar

$\phi_{\alpha}(\mathbf{P}) \simeq \phi_{\alpha}\left(\mathbf{P}^{o}\right)+\Delta \mathbf{P}^{T}\left(\mathbf{g}_{d}+\alpha \mathbf{g}_{r}\right)+\frac{1}{2} \Delta \mathbf{P}^{T}\left(\mathbf{H}_{d}+\alpha \mathbf{H}_{r}\right) \Delta \mathbf{P}$

em que considerou-se

$$
\begin{aligned}
\mathbf{H}_{d} & =\left.\left(\nabla_{\mathbf{P}} \nabla_{\mathbf{P}}^{T} \phi_{d}(\mathbf{P})\right)\right|_{\mathbf{P}=\mathbf{P}^{o}} \\
\mathbf{H}_{r} & =\left.\left(\nabla_{\mathbf{P}} \nabla_{\mathbf{P}}^{T} \phi_{r}(\mathbf{P})\right)\right|_{\mathbf{P}=\mathbf{P}^{o}} \\
\mathbf{g}_{d} & =\left.\left(\nabla_{\mathbf{P}} \phi_{d}(\mathbf{P})\right)\right|_{\mathbf{P}=\mathbf{P}^{o}} \\
\mathbf{g}_{r} & =\left.\left(\nabla_{\mathbf{P}} \phi_{r}(\mathbf{P})\right)\right|_{\mathbf{P}=\mathbf{P}^{o}}
\end{aligned}
$$

Logo, para se ter uma estimativa de $\mathbf{P}$ que minimize (8), se pode aplicar o operador gradiente $\nabla_{\mathbf{P}}$ na mesma e igualar o resultado desta operação ao vetor nulo (Régis, 2000), de modo a obter a seguinte equação normal

$$
\left[2 \mathbf{A}^{T} \mathbf{A}+\alpha \mathbf{H}_{r}\right] \Delta \mathbf{P}=2 \mathbf{A}^{T}\left[\mathbf{d}^{o}-\mathbf{f}\left(\mathbf{P}^{o}\right)\right]-\alpha \mathbf{g}_{r},
$$

em que $\mathbf{H}_{r}$ e $\mathbf{g}_{r}$ serão determinados de acordo com a definição do funcional regularizador. A matriz $\mathbf{A}$, denominada matriz de sensibilidade dos dados em relação aos parâmetros, possui seus componentes definidos pela igualdade

$$
A_{i j}=\left.\frac{\partial f_{i}(\mathbf{P})}{\partial P_{j}}\right|_{\mathbf{P}^{o}}
$$

sendo que neste trabalho a aproximaremos da seguinte maneira

$$
A_{i j}=\left.\frac{\partial f_{i}(\mathbf{P})}{\partial P_{j}}\right|_{\mathbf{P}^{o}} \simeq \frac{f_{i}\left(\mathbf{P} ; P_{j}^{o}+\delta P_{j}^{o}\right)-f_{i}\left(\mathbf{P} ; P_{j}^{o}-\delta P_{j}^{o}\right)}{2 \delta P_{j}^{o}},
$$

sendo $\delta P_{j}^{o}$ uma pequena pertubação do valor do parâmetro $P_{j}^{o}$, que costuma ser um percentual pequeno do valor do mesmo. Neste trabalho será utilizado o valor de 5 $\%$.

A técnica de Marquardt (Marquardt, 1963), busca garantir a estimativa de um passo sempre na direção descendente do gradiente de $\phi_{\alpha}(\mathbf{P})$. Isto é feito alternando entre a estimativa com o m'etodo de Gauss-Newton, equação (13), e com um pequeno passo na direção descendente do gradiente de $\phi_{\alpha}(\mathbf{P})$, semelhante ao método de gradiente descendente, ou Method of Steepest Descent Arfken (1985).

Englobando estas duas formas de buscar um mínimo para a função objetivo, em uma única equação, acrescentase um escalar positivo $\lambda$, denominado parâmetro de Marquardt, em (13), somando-o a diagonal da matriz hessiana dos funcionais, ou seja,

$$
\left[\mathbf{A}_{k}^{T} \mathbf{A}_{k}+\alpha \mathbf{H}_{r k}+\lambda \mathbf{I}\right] \Delta \mathbf{P}_{k}=\mathbf{A}_{k}^{T}\left[\mathbf{d}^{o}-\mathbf{f}\left(\mathbf{P}_{k}\right)\right]-\alpha \mathbf{g}_{r k},
$$

em que o valor de $\lambda$ aumenta ou diminui durante as iterações de modo a deixar a matriz hessiana sempre diagonal dominante.

Neste trabalho serão adotados os seguintes critérios de convergência:

1. Quando os dados já estiverem ajustados dentro de uma certa precisão, equação (17), e não houver variação significativa da função objetivo, equação (18), ou seja, quando forem satisfeita a condições

$$
\left\|\mathbf{d}^{o}-F(\mathbf{P})\right\|^{2} \leq \delta
$$

e

$$
\left|\frac{\phi_{\alpha}\left(\mathbf{P}_{k-1}\right)-\phi_{\alpha}\left(\mathbf{P}_{k}\right)}{\phi_{\alpha}\left(\mathbf{P}_{k}\right)}\right|<\varepsilon
$$

sendo $\delta$ a raíz quadrada da média quadrática dos ruídos aleatórios contidos nos dados e $\varepsilon$ um escalar positivo de valor bem pequeno.

2. Estando os dados ajustados, se em uma dada iteração $k$ não houver variação considerável dos valores dos parâmetros estimados, com relação a iteração anterior, ou seja, quando for satisfeita a condição

$$
\left\|\mathbf{P}_{k}-\mathbf{P}_{k-1}\right\|^{2} \leq \gamma,
$$

com $\gamma$ sendo um escalar maior que zero e de valor pequeno.

3. Se o número máximo de iterações, pré definido, for atingido.

\section{Regularizador Suavidade Global}

O vínculo de suavidade global (SG), sujeito ao ajuste dos dados, faz com que o processo convirja para uma solução em que as diferenças entre os valores dos parâmetros sejam mínimas, ou melhor, uma solução em que não há variações abruptas entre os valores dos parâmetros. 
A representação matemática do funcional SG pode ser escrita como

$$
\phi_{S G}(\mathbf{P})=\sum_{k=1}^{N d}\left|\left(P_{i}-P_{j}\right)_{k}\right|^{2}, \quad i \neq j, \text { i e } j=1,2, \ldots, N p,
$$

ou mesmo na forma matricial

$$
\phi_{S G}(\mathbf{P})=\|\mathbf{S P}\|^{2},
$$

em que $N d$ é o número de diferênças entre os parâmetros, $N p$ o número de parâmetros e $\|.\|^{2}$ a norma dois, ou euclidiana ${ }^{1}$, ao quadrado, do vetor de diferenças SP, onde a matriz $\mathbf{S}$ guarda as informações de como os parâmetros se relacionam. Sua estrutura é de tal forma que, quando multiplicada ao vetor de parâmetros, gere um vetor de diferenças entre parâmetros que se quer relacionar. Para ilustrar a construção de $\mathbf{S}$, tal qual como foi montada para este trabalho, considera-se dois exemplos com seis parâmetros. No primeiro se quer um vetor que guarde a diferença entre os parâmetros adjacentes na horizontal

$$
(\mathbf{S P})^{T}=\left[P_{1}-P_{2}, P_{2}-P_{3}, P_{3}-P_{4}, P_{4}-P_{5}, P_{5}-P_{6}\right] .
$$

no qual o arranjo é mostrado na figura (1).

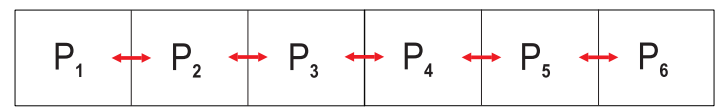

Figure 1: Arranjo dos parâmetros em uma estrutura 1D, que pode ser tanto na vertical como na horizontal.

Para o cado da figura 1, a matriz $\mathbf{S}$ é dada por

$$
\mathbf{S}=\left[\begin{array}{cccccc}
1 & -1 & 0 & 0 & 0 & 0 \\
0 & 1 & -1 & 0 & 0 & 0 \\
0 & 0 & 1 & -1 & 0 & 0 \\
0 & 0 & 0 & 1 & -1 & 0 \\
0 & 0 & 0 & 0 & 1 & -1
\end{array}\right]
$$

A matriz hessiana e o vetor gradiente do funcional de suavidade global, após algumas manipulações matemáticas, serão determinadas por

$$
\begin{aligned}
\mathbf{g}_{S G} & =\nabla_{\mathbf{P}} \phi_{S}(\mathbf{P})=2 \mathbf{S}^{T} \mathbf{S P} \\
\mathbf{H}_{S G} & =\nabla_{\mathbf{P}} \nabla_{\mathbf{P}}^{T} \phi_{S}(\mathbf{P})=2 \mathbf{S}^{T} \mathbf{S}
\end{aligned}
$$

\section{Regularizador Minimum Gradient Support}

O regularizador Minimum Gradient Support (MGS) é usado para soluções com descontinuidades nos valores de alguns parâmetros (Zhang et al., 2012), enquanto que para outros há apenas variação suave entre seus valores.

\footnotetext{
${ }^{1} \mathrm{~A}$ norma $n$ de um vetor pode ser determinada por $\|\mathbf{u}\|_{n}=$ $\left[\sum_{i=1}^{N}\left|u_{i}\right|^{n}\right]^{\frac{1}{n}}$.
}

Pode-se definir o funcional do vínculo MGS da seguinte maneira

$$
\begin{aligned}
& \phi_{M G S}(\mathbf{P})=\sum_{k=1}^{N d} \frac{\left(P_{i}-P_{j}\right)_{k}^{2}}{\left(P_{i}-P_{j}\right)_{k}^{2}+\beta^{2}}, \\
& i \neq j, \text { i e } j=1,2, \ldots, N p,
\end{aligned}
$$

sendo $\beta$ um escalar real de valores pequenos, porém maiores que zero. O critério para a escolha do valor dessa constante é testar o menor valor que combinado com o valor ótimo do parâmetro de regularização, possibilite uma solução estável para o problema. Definido o funcional, deve-se obter seu vetor gradiente bem como sua matriz hessiana, de modo que com alguns cálculos pode-se encontrar

$$
\mathbf{G}_{M G S}=2 \mathbf{S}^{T} \mathbf{W S P}
$$

e também

$$
\mathbf{H}_{M G S}=2 \mathbf{S}^{T} \mathbf{W S},
$$

sendo $W$ uma matriz diagonal, com seus valores não nulos dados por

$$
W_{k k}=\frac{\beta^{2}}{\left[\left(P_{i}-P_{j}\right)_{k}^{2}+\beta\right]^{2}} .
$$

\section{Resultados}

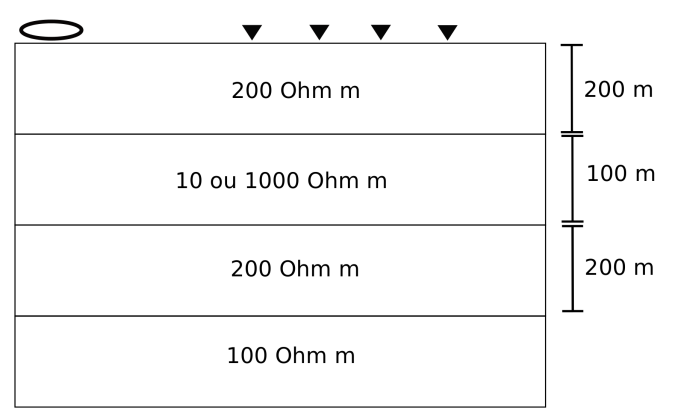

Figure 2: Modelo esquemático do meio estratificado que gerou os dados para a inversão.

Para testar o algorítimo de inversão desenvolvido neste trabalho, foram gerados dados do DMV para dois modelos de camadas diferentes, um com uma camada que é mais condutiva que o meio a qual está encaixada e outro no qual a camada é mais resistiva que o meio no qual está presente. O primeiro modelo consiste de quatro camadas, sendo a última o embasamento. A primeira posui uma resistividade de $200 \Omega \mathrm{m}$ e espessura de $200 \mathrm{~m}$, a segunda tem resistividade de $10 \Omega \mathrm{m}$ e espessura de $100 \mathrm{~m}$, a terçeira resistividade de $200 \Omega \mathrm{m}$ e espessura de $200 \mathrm{~m}$ e a última a resistividade de $100 \Omega m$ e espessura infinita. O modelo dois possui as mesmas características do primeiro, porém somente a resistividade da segunda camada que é diferente, no primeiro modelo a resistividade é de $10 \Omega \mathrm{m}$ e no modelo dois $1000 \Omega \mathrm{m}$. Um exemplo esquemático dos modelos pode ser visualizado na figura 2. Para os dois modelos, calculou-se a componente radial do campo magnético $\bar{H}_{r}$ para 20 posições de receptores, que vão de 

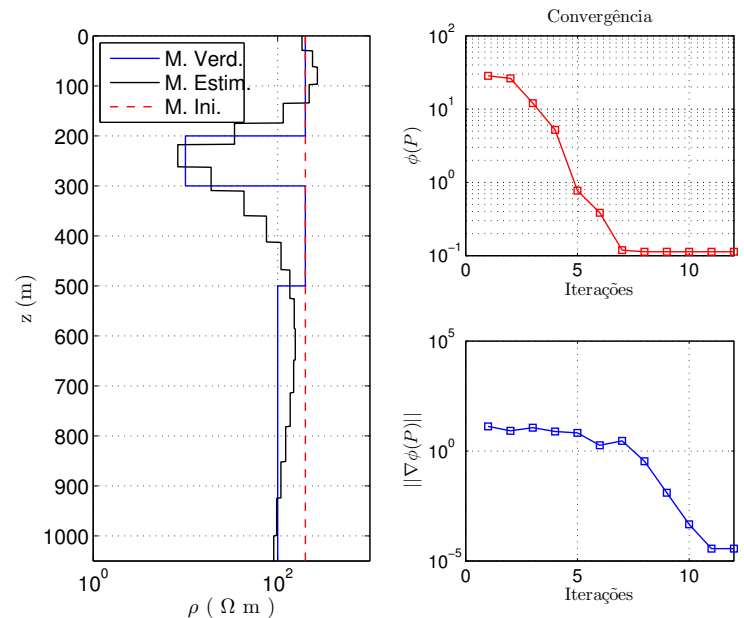

Figure 3: Resultado 1D com o vínculo SG para $\alpha=10^{-2}$.
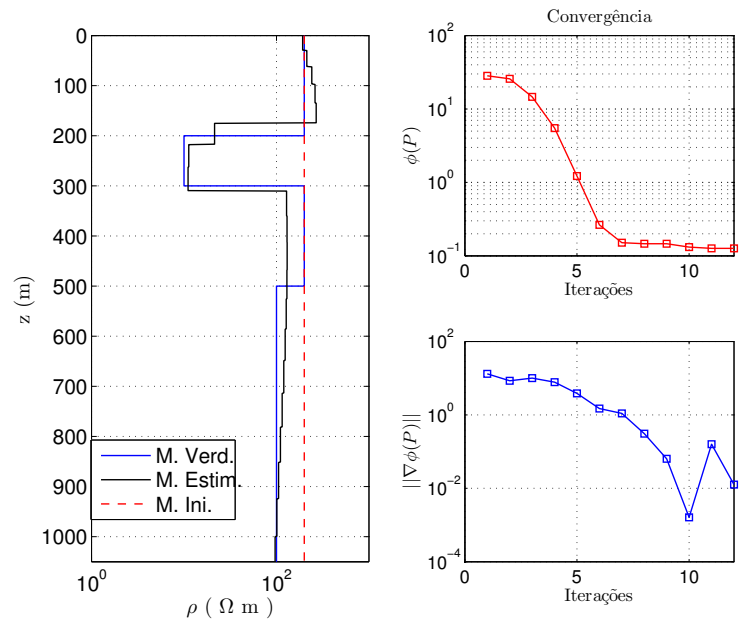

Figure 4: Resultado 1D com o vínculo MGS para $\alpha=10^{-2}$.

$100 \mathrm{~m}$ até $2 \mathrm{~km}$ do centro da bobina que tem um raio de $5 \mathrm{~m}$ e 21 frequências, na faixa de $0.001 \mathrm{~Hz}$ a $1 \mathrm{kHz}$. Para o caso em que se tem a camada alvo mais condutiva que 0 meio encaixante gerou-se resultados de inversão utilizando os vínculos SG e MGS, mostrados nas figuras 3 e 4, usando o parâmetro de regualrização igual a $\alpha=10^{-2}$ nos dois casos. No caso em que se tem a camada mais resistiva que o meio encaixante, gerou-se resultados para os mesmos regularizadores mencionados anteriormente. Nos dois casos, o parâmetro de regularização utilizado foi $\alpha=10^{-2}$. Os modelos interpretativos recuperados podem ser vistos nas figuras 5 e 6 .

\section{Conclusões}

Os resultados apresentados pelos modelos interpretativos, a partir da inversão, mostram que os regularizadores SG e MGS identificaram melhor as camadas alvos condutivas, se comparado com as resistivas, o que pode ser esperado devido os dados do DMV serem bastante influenciados por efeitos indutívos. Os regularizadores utilizados nos resultados de inversão mostraram-se
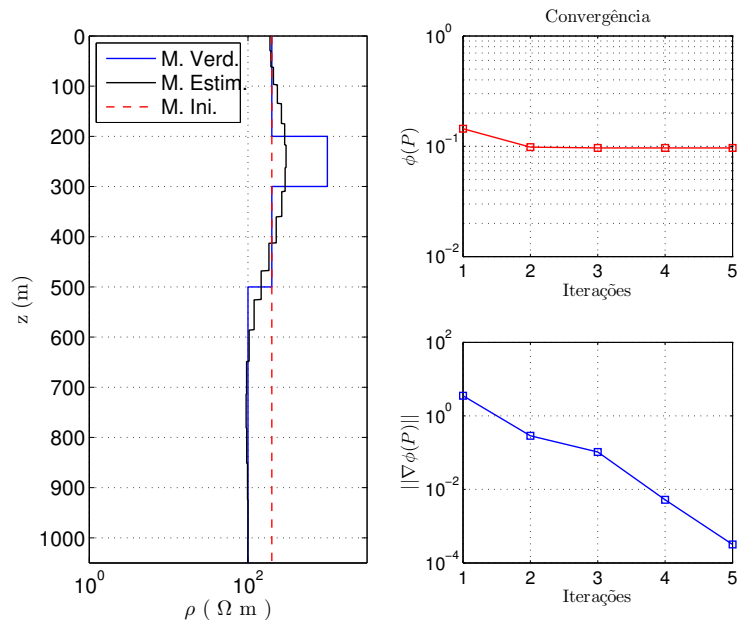

Figure 5: Resultado 1D com o vínculo SG para $\alpha=10^{-2}$.
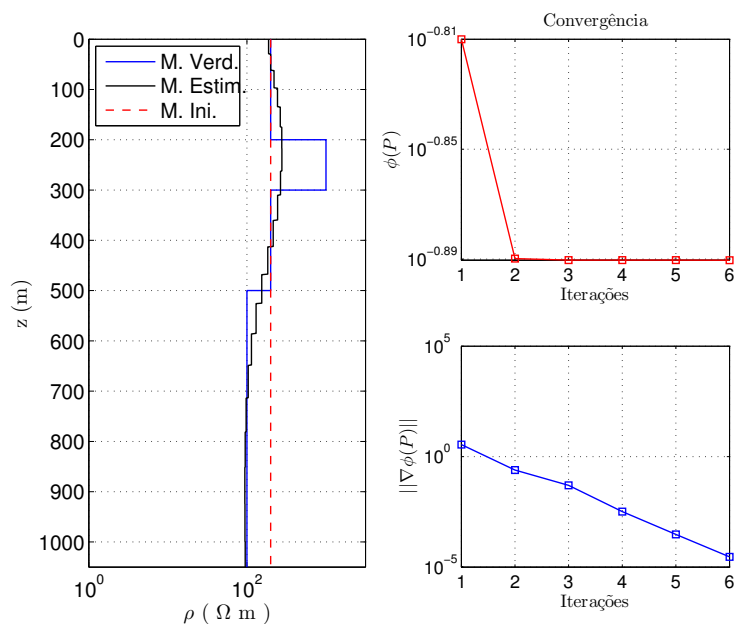

Figure 6: Resultado 1D com o vínculo MGS para $\alpha=10^{-2}$.

bastante eficientes na busca de soluções estáveis e únicas para os modelos propostos, podendo o algorítimo desenvolvido neste trabalho ser utilizado com dados reais com bastante confiabilidade, com a finalidade de uma melhor interpretação do meio geológico.

\section{Agradecimentos}

À Universidade Federal do Oeste do Pará e ao corpo docente da instituição, à SBGf pela oportunidade de divulgação das pesquisas desenvolvidas, aos laboratórios LAMGEO e LIGEO pela disponibilidade de espaço, e ao orientador por ceder computadores para o desenvolvimento do trabalho.

\section{Referências}

Arfken, G., 1985, Mathematical methods for physicists, third ed.: Academic Press.

Marquardt, D. W., 1963, An algorithm for least-squares estimation of nonlinear parameters: SIAM Journal on Applied Mathematics, 11, 431-441.

Philip Kearey, Michael Brooks, I. H., 2009, Geofísica de 
exploração: Oficina de textos.

Piedade, A. A., and C. R. T. Régis, 2014, Inversion of data from the multifrequency eletromagnetic method.: Presented at the Seg International Exposition and 84th Annual Meeting., SEG, SEG.

Régis, C. R. T., 2000, Inversão vinculada de dados de emap e mt-1d anisotrópico: Tese de doutorado, Programa de Pós-Graduação em Geofísica, Universidade Federal do Pará.

Rijo, L., 2004, Eletrical geophysics 1-d earth modeling.

Zhang, L., T. Koyama, H. Utada, P. Yu, and J. Wang, 2012, A regularized three-dimensional magnetotelluric inversion with a minimum gradient support constraint: Geophysics, 296-316. 